\title{
Nuevo registro para México de Gleditsia (Leguminosae)
}

Gleditsia L. es un género de árboles que usualmente presentan espinas ramificadas; en la mayoría de sus especies sus hojas alternas son de dos tipos: una o dos veces pinnadas. Se trata de especies con flores unisexuales o polígamas que producen una legumbre indehiscente, linear u oval, larga o corta y con varias a una sola semilla.

Es un género con aproximadamente 14 especies, conocido en Asia y representado en América por dos especies en Estados Unidos y otra en Sudamérica (Robertson y Lee, 1976; Burkart, 1952).

Al revisar las colecciones de Gleditsia depositadas en los herbarios MEXU y ENCB y la literatura sobre su distribución, se encontró que parece ser el primer registro en estado silvestre de este género para la flora de México.

Gleditsia triacanthos L., Sp. Pl. 1056. 1753. Es una especie nativa y cultivada en Estados Unidos, a la cual pertenecen los ejemplares colectados. Su distribución general, según Vines (1984), es: Texas, Oklahoma, Arkansas y Luisiana; al este a Florida, al norte a Pensilvania y Nueva York y al oeste a Nebraska.

El nuevo registro para México se encontró en: Tamaulipas, cresta y exposición sur del Cerro Bufa El Diente, Sierra de San Carlos; altitud 1400 msnm, sobre sustrato ígneo. Colectas: O. Briones 1187 (11-X-1984), 1748 (23-V-1985), 1795 (15-VI-1985), 2209 (23-IV-1986: flores amarillo-verdoso).

Los ejemplares se encuentran depositados en los herbarios ENCB, ANSM, y MEXU.

En la cresta del cerro es el árbol más importante, ya que contribuye con $91 \%$ de la densidad total (1 487 ind. / ha) y en área basal alcanza el valor más alto (57\% del total) gracias a su alta densidad, ya que sus troncos suelen ser más bien delgados. Junto con G. triacanthos crecen: Carpinus caroliniana Walt. y Quercus sartorii Lieb.

\begin{tabular}{|c|c|c|c|c|c|c|}
\hline \multirow[b]{2}{*}{ Especies } & \multicolumn{2}{|c|}{ Densidad } & \multirow{2}{*}{$\begin{array}{l}\text { Diámetro } \\
\text { medio / cm }\end{array}$} & \multirow{2}{*}{$\begin{array}{c}\text { Altura } \\
\text { media } / m\end{array}$} & \multicolumn{2}{|c|}{ Área basal } \\
\hline & No / ba & $\%$ & & & $m^{2} / b a$ & $\%$ \\
\hline Gleditsia triacanthos & 1487 & 91 & 9.0 & 6.4 & 9.7 & 57 \\
\hline Carpinus caroliniana & 103 & 6 & 16.7 & 6.0 & 2.4 & 14 \\
\hline Quercus sartorii & 51 & 3 & 35.2 & 8.4 & 4.9 & 29 \\
\hline Total & 1641 & 100 & 10.3 & 6.5 & 17.0 & 100 \\
\hline
\end{tabular}

G. aquatica Marshall es un árbol similar a G. triacanthos. Crece en la planicie costera del sureste de Estados Unidos: Carolina del Norte, centro-sur de Florida y extremo 
este de Texas. Para el área en que las dos especies se traslapan se ha señalado un híbrido: Gleditsia $\times$ texana Sarg. (Elías, 1980).

G. aquatica muestra preferencias por lugares más húmedos de tipo ripario. Aunado a las diferencias en hábitat y distribución entre G. triacanthos y G. aquatica, la diferencia morfológica más evidente radica en los frutos. En la primera especie son largos, aplanados y con muchas semillas, mientras que en la segunda son mucho más pequeños, ovales y con 1-3 semillas. Los ejemplares de G. triacanthos colectados en Tamaulipas no presentan frutos; sin embargo, fue posible ubicarlos en dicho taxón gracias a otros caracteres morfológicos: corteza gruesa y rugosa, espinas generalmente 3-partidas y hojas compuestas con 4-7 pares de pínnulas.

AgRADECIMIENTOS. Se agradece al Dr. Burkhard Müller-Using su colaboración en el levantamiento forestal y al Dr. Jorge S. Marroquín de la Fuente el aporte bibliográfico y la revisión crítica del manuscrito.

\section{LITERATURA CITADA}

Burkart, A., 1952. Las leguminosas argentinas silvestres y cultivadas. ACME Agency. Buenos Aires, pp. 185-187. ELÍAS, T.S., 1980. The complete trees of North America. Field guide and natural history. Ed. Van Nostrand Reinhold Co. New York, pp. 648-650.

RoberTSON, K.R. y YIN-TSE LEE, 1976. The genera of Caesalpinioideae (Leguminosae) in the Southeastern United States. J. Arnold Arbor. 57(1):1-53.

VINES, R.A., 1984. Trees, shrubs and woody vines of the Southwest. University of Texas Press, Austin. 1104 pp.

ÓSCAR L. Briones V. Facultad de Silvicultura y Manejo de Recursos Renovables, U.A.N.L. 